sacroespinosa bilateral de la cúpula a 48 mujeres y 40 a mujeres se les practicó una aproximación abdominal con suspensión colposacra y reparo paravaginal. Se realizaron procedimientos auxiliares cuando estaban indicados. Se hicieron exámenes pélvicos cuidadosos en el postoperatorio por parte del coautor no cirujano, cada año hasta 5 años. Las mujeres se examinaron en posición de pie durante un esfuerzo máximo. La cirugía se clasificó como óptimamente efectiva cuando la mujer permanecía asintomática, el apex vaginal estaba sostenido por encima de la placa del elevador y no ocurrió ninguna protrusión de tejido vaginal mas allá del himen. La efectividad quirúrgica se consideró como insatisfactoria si la mujer estaba sintomática, el apex descendía $>50 \%$ de su longitud o la pared vaginal protruía mas allá del himen.

Resultados: Ochenta mujeres (42 del grupo vaginal, 38 del grupo abdominal) estuvieron disponibles para la evaluación entre el año y los 5.5 años (promedio 2.5 años). Los grupos fueron comparables en edad, peso, paridad y estado estrogénico, y $56 \%$ habían tenido una cirugía pélvica anterior. No hubo diferencia significativa entre los grupos en morbilidad, complicaciones, cambio en la hemoglobina, dispareunia, dolor o estancia hospitalaria. El grupo vaginal tuvo mayor duración de uso del catéter, mas infecciones del tracto urinario, mas incontinencia, menor tiempo operatorio y menor costo hospitalario. La efectividad quirúrgica fue óptima en el $29 \%$ del grupo vaginal y $58 \%$ del grupo abdominal y fue insatisfactoria requiriendo reoperación en un 33\% del grupo vaginal y 16\% del grupo abdominal. Las reoperaciones incluyeron procedimientos para incontinencia urinaria recurrente en el 12\% del grupo vaginal y $2 \%$ del grupo abdominal. El RR de efectividad óptima por la vía abdominal es 2.03 (IC 95\% 1.22 a 9.83) y el RR de resultado insatisfactorio usando la vía vaginal es 2.11 (IC $95 \% 0.90$ a 4.94 ).

Conclusiones: La cirugía pélvica reconstructiva para la corrección de los defectos significativos del soporte pélvico fue mas efectiva con la aproximación abdominal.

\section{Histerectomía después de la ablación endometrial}

\author{
James B. Unger, MD, and G. Rodney Meeks, MD
}

From the Department of Obstetrics and Gynecology, Marshfield Clinic, and the Department of Obstetrics and Gynecology, University of Mississippi Medical Center

Am J Obstet Gynecol 1996; 175: 1432-1437.

Objetivos: Determinar el número de mujeres a quienes se les practica histerectomía después de la ablación endometrial y las indicaciones para la cirugía subsecuente.

Diseño del estudio: Se practicó ablación endometrial con esfera a 42 mujeres premenopáusicas quienes tenían severa menorragia asociada con un resultado normal del examen clínico, entre noviembre de 1990 y diciembre de 1991. Se evaluaron 37 mujeres a quienes se les ha hecho cuidado continuo revisando la historia clínica. Se entrevistaron por teléfono cuatro mujeres quienes recibieron cuidado en otra localidad. Una mujer se perdió del seguimiento. Las pacientes fueron seguidas por un mínimo de 4 años. Se analizaron la edad, paridad, tiempo operatorio, prepara- ción endometrial, esterilización preablación y dismenorrea preablación con respecto a la histerectomía subsecuente. La satisfacción de la paciente se evaluó a los 24 meses. Se hicieron análisis de tablas de vida para determinar la probabilidad acumulativa de histerectomía.

Resultados: Se practicó histerectomía a catorce de las 41 mujeres (34\%) dentro de los 5 años siguientes a la ablación endometrial con esfera. La hemorragia menstrual anormal continuada y el dolor menstrual se asociaron significativamente con la histerectomía subsecuente. Once de los 14 casos de histerectomía se asociaron con anormalidad macroscópica tales como miomas, adenomiosis, endometriosis y hematosálpinx crónico. Se observó una relación linear entre la histerectomía y el tiempo.

Conclusión: Con base en los hallazgos un tercio de las mujeres a quienes se les practica ablación endometrial con esfera para menorragia pueden esperar que se les practique histerectomía dentro de los 5 años siguientes. Si la relación linear observada durante los primeros cinco años se extrapola, teóricamente, todas las mujeres necesitarán histerectomía alrededor de los 13 años. La mayoría de las pacientes requirieron histerectomía por anormalidades pélvicas significativas. Se requieren más estudios con seguimiento a largo plazo para definir el papel de la ablación endometrial para la menorragia.

\section{El papel de la colpocleisis con alargamiento uretral en la faloplastia transexual}

Ralph R. Chesson, MD, ${ }^{\mathrm{c}}$ David A. Gilbert, MD, ${ }^{\mathrm{a}}$ Gerald H. Jordan, MD, ${ }^{b}$ Steven M. Schlossberg, MD, ${ }^{b}$ Gerald T. Ramsey, $\mathrm{PhD}$, and Deborah M. Gilbert, $\mathrm{RN}^{\mathrm{a}}$

\section{From the Department of Plastic Surgery, ${ }^{a}$ Urology, ${ }^{b}$ and Obstetrics and Gynecology, ${ }^{c}$ The Eastern Virginia Medical School}

\section{Am J Obstet Gynecol 1996; 175: 1443-1450.}

Objetivo: La cirugía transexual es un área única de intervenciones poco frecuentes. Este estudio examina los factores que tienen significancia en la prevención de la morbilidad mayor en esta cirugía inusual. Se presenta el papel del ginecólogo en el manejo psicológico, endocrino y quirúrgico.

Diseño del estudio: Las cirugías iniciales se complicaron con fístulas en el sitio de la anastomosis uretra-falo. Después de revisar estas complicaciones, modificamos la aproximación incluyendo un procedimiento de dos etapas permitiendo la curación antes de la microcirugía y preservando la pared vaginal anterior durante la histerectomía vaginal y colpocleisis. Preservando la pared vaginal anterior, podemos extender mejor la uretra para unión posterior al falo.

Resultados: Realizando el procedimiento en dos etapas en la colpocleisis se permite una reducción significativa en la tasa de fístulas $(\mathrm{p}=0.0087)$. Con la eliminación de las fístulas, es posible el uso de endurecedores durante la faloplastia para mejores resultados funcionales.

Conclusión: La extensión de la uretra durante la colpocleisis permite una curación mejor y disminuye significativamente la formación de fístulas. Un flujo sanguí- 
neo apropiado por cirugía microvascular y un tejido adecuado para el sitio de anastomosis contribuye a mejorar los resultados.

\section{Tres clases de histerectomía vaginal radical para el tratamiento del cáncer endometrial y cervical}

Giambattista Massi, MD, Luciano Savino, MD, and Tommaso Susini, MD

\section{From the Obstetrics and Gynecology Department, University of Florence}

Am J Obstet Gynecol 1996; 175: 1576-1585.

Objetivos: Los propósitos fueron: (1) describir tres tipos de histerectomía vaginal extendida con diferentes grados de radicalidad, (2) identificar posibles indicaciones para cada una de ellas y (3) estimular la individualización del tratamiento, con especial referencia a la reevaluación del papel de la cirugía vaginal en la oncología ginecológica.

Diseño del estudio: Se ilustran los principios quirúrgicosanatómicos de la cirugía vaginal radical y las técnicas de tres histerectomías vaginales con extensión en incremento. Las posibles indicaciones se presentan con base en la experiencia propia de estudios retrospectivos publicados previamente.

Resultados: La clase I de histerectomía vaginal extendida permite la disección «en bloque» del útero junto con el tercio superior de vagina y ambos anexos. No se remueven los parametrios. Este procedimiento ha demostrado ser valioso para el tratamiento del cáncer endometrial Estado I. En la clase II de histerectomía vaginal extendida se preserva el tracto distal de los parametrios anterior y posterior, mientras que se remueve completamente el ligamento cardinal. Esta operación ha mostrado resultados prometedores para el tratamiento del cáncer cervical estados IB-IIA de pequeño volumen mientras que reduce la incidencia de disfunciones vesicales y rectales. Los procedimientos de clase III incluyen la remoción completa de los parametrios (anterior, lateral y posterior). Esta operación ha mostrado que proporciona una alta tasa de curación para el cáncer cervical estados IB-IIA.

Conclusiones: En vista de las múltiples ventajas de la cirugía vaginal, ésta aproximación debe ser considerada para el tratamiento individualizado de casos seleccionados de cánceres endometrial y cervical. Las tres clases de histerectomía vaginal permiten ajustar el tipo de operación a las características clínicas y físicas de las pacientes. El uso combinado de la linfadenectomía extraperitoneal o laparoscópica podrían extender considerablemente las indicaciones para las operaciones vaginales radicales.

\section{Influencia relativa de la edad y la menopausia en los cambios de la composición corporal total y regional en mujeres postmenopáusicas}

Florence A. Trémolliers, $\mathrm{MD}, \mathrm{PhD}$, Jean-Michel Pouilles, $\mathrm{MD}$, and Claude A. Ribot, MD

From the Unité Fonctionnelle Ménopause et Maladies Métaboliques, Service d'Endocrinologie, Centre Hospitalier Universitaire Purpan

Am J Obstet Gynecol 1996; 175: 1594-1600.
Objetivo: Se midió la composición corporal total y regional para evaluar las diferencias en la composición asociadas con la menopausia y para determinar si los cambios en la distribución de la grasa estaban más relacionadas con la edad o con la menopausia.

Diseño del estudio: Se estudiaron 205 mujeres blancas saludables quienes no habían recibido terapia de reemplazo estrogénico de acuerdo con el estado menopáusico y la edad. La masa ósea y la composición corporal se midieron por absorciometría dual con rayos X. Se calcularon las proporciones de grasa androide y ginecoide en todas las mujeres y se investigaron las diferencias por análisis estadísticos.

Resultados: Comparadas con las mujeres premenopáusicas, las mujeres posmenopáusicas se caracterizaron por un aumento significativo en la proporción de grasa androide y en la relación grasa del tronco/grasa de la pierna, mientras que la cantidad absoluta de masa corporal grasa no cambia significativamente. Las diferentes variables de distribución androide de la grasa tienden a correlacionarse mejor con los años desde la menopausia que con la edad. En el análisis de regresión múltiple, los años desde la menopausia fueron un predictor de la masa corporal grasa y grasa del tronco, mientras que la edad no fue predictora de ninguna de las variables de distribución de la grasa.

Conclusiones: Este estudio subraya los cambios tempranos en la distribución de la grasa corporal con una desviación de la grasa corporal hacia una localización mas central en la mujer posmenopáusica. Este cambio en la distribución de la grasa parece estar mas relacionado con la menopausia que con la edad y podría, junto con otros factores, contribuir a explicar el aumento del riesgo cardiovascular informado en mujeres posmenopáusicas.

\section{Respuesta inmune específica contra hemolisina de Gardnerella vaginalis en pacientes con vaginosis bacteriana}

Sabina Cauci, $\mathrm{PhD},{ }^{\mathrm{a}}$ Federica Scrimin, MD, ${ }^{\mathrm{b}}$ Silvia Driussi, MD, ${ }^{c}$ Sandro Ceccone, MS, ${ }^{a}$ Rossella Monte, MS, ${ }^{a}$ Loris Fant, MS, ${ }^{\mathrm{d}}$ and Franco Quadrifoglio, $\mathrm{PhD}^{\mathrm{a}}$

From the Department of Biomedical Sciences and Technologies, School of Medicine, University of Udine, ${ }^{a}$ the Institute of Obstetrics and Gynecology, Istituto di

Ricovero e Cura a Carattere Scientifico "Burlo

Garofolo," Azienda Sanitaria Locale 4, " and the Microbiology Unit, San Daniele Hospital ${ }^{d}$

Am J Obstet Gynecol 1996; 175: 1601-1605.

Objetivo: Estudiar la respuesta mucosa del hospedero en vaginosis bacteriana evaluando la presencia de una respuesta inmune específica contra la hemolisina deGardnerella vaginalis en fluidos vaginales de pacientes y verificar su correlación con los criterios adoptados para diagnosticar la vaginosis bacteriana.

Diseño del estudio: Se incluyeron un total de 123 mujeres blancas que consultaron a una unidad de cuidado ginecológico por quejas urogenitales o para tamizaje de cáncer uterino (prueba de Papanicolaou), con edades entre 20 y 60 años y no menstruando. La vaginosis bacteriana se diagnosticó por criterios clínicos y un puntaje de la tinción de Gram $>6$. 